\title{
O contributo da experiência turística para o desenvolvimento do turismo nos destinos rurais: uma revisão da literatura
}

\author{
Bárbara Manuela de Jesus Mateiro* \\ Universidade de Aveiro (Portugal)
}

\begin{abstract}
Resumo: As áreas rurais têm vindo a ganhar outra dinâmica económica, social e cultural, que resulta, não só, mas também da prática turística. Principalmente, a partir do novo milénio, houve a necessidade dos destinos se adaptarem a novas modalidades turísticas, como é o caso do turismo rural, que privilegiam a pequena escala, o contacto direto com as comunidades locais, bem como com os recursos naturais e culturais. Isto resulta da alteração que ocorreu no comportamento dos visitantes, onde estes são cada vez mais sensíveis às experiências turísticas. Tais factos salientam a importância de compreender e mensurar a experiência turística, assim como o seu impacte no desenvolvimento sustentável dos destinos rurais. Deste modo, com base numa revisão da literatura publicada nas últimas três décadas e meia, procurou-se compreender o estado da arte da experiência turística, identificando as dimensões que lhe têm vindo a ser atribuídas. Adicionalmente pretendeu-se perceber como estas podem contribuir para o desenvolvimento turístico das áreas rurais. Os resultados evidenciam que a experiência turística tem diferentes dimensões que foram evoluindo e que variam de acordo com diferentes autores. Esta dificuldade de mensuração leva a que seja igualmente difícil perceber o verdadeiro impacte que as experiências turísticas possuem no desenvolvimento do turismo nas áreas rurais.
\end{abstract}

Palavras-chave: Economia de experiencias; Experiência turística; Desenvolvimento turístico; Áreas rurais; Turismo rural; Revisão da literatura.

The contribution of tourism experience to the development of tourism in rural destinations: a literature review

Abstract: Rural areas have taken on new economic, social and cultural dynamism, as a result, not exclusively, but to a large extent, of the development of tourism. From the beginning of the 21st century, destinations have had to adapt to new forms of tourism, such as rural tourism, with all of its advantages such as small scale and direct contact with local communities as well as natural and cultural resources. This is a result of the change that has occurred in the behaviour of visitors, who tend to quest experiences rather than consumption of place. These facts underscore the importance of understanding and measuring tourist experience, as well as its impact on the sustainable development of rural destinations. Thus, based on a review of the relevant literature of the last three and a half decades, we aim at understanding the state of the art in tourism experiences, and to dimensions the parameters by which these are measured.. Additionally, we attempt to grasp how tourism experiences feed into the development of tourism in rural areas. The results show that tourism experiences mean different things to different people. The difficulties encountered in applying quantitative measurement make it equally complicated to gauge the true impact that tourism experiences have on the development of tourism in rural areas.

Keywords: Experience economy; Tourist experience; Tourist development; Rural areas; Rural tourism; Literature review. 


\section{Introdução}

A atual sociedade é composta por indivíduos que desejam não apenas satisfazer as suas necessidades básicas através da aquisição de bens e serviços, mas também vivenciar experiências únicas e marcantes. Segundo Pine e Gilmore (1998), as experiências representam a quarta fase do ciclo da progressão do valor económico. Quando se transporta esta realidade para o Turismo verifica-se que principalmente a partir do século XXI, este setor passou a estar fortemente ligado ao fornecimento e gestão de experiências (Agapito, Valle \& Mendes, 2012). Repare-se que isto resulta da evolução das alterações no comportamento dos visitantes, já que estes estão mais predispostos a vivenciar experiências (Swarbrooke \& Horner, 2002). Contudo, para que a experiência seja única e memorável, os destinos turísticos devem planear, criar, desenvolver e monitorizar ambientes propícios ao desenvolvimento de experiências turísticas, pois estas contêm um conjunto de recursos valiosos para os lugares e geram valores emocionais, cognitivos e comportamentais nos visitantes (Mossberg, 2007; Schmitt, 1999).

Neste seguimento de ideias tem-se vindo a observar um crescente interesse nesta temática, não apenas por parte dos académicos, mas também por parte dos empresários que desejam cada vez mais desenvolver experiências que possam marcar positivamente os visitantes (Walls, Okumus, Wang \& Kwun, 2011). Deste modo, acredita-se que perceber e mensurar a experiência turística deve ser o primeiro passo para a criação e desenvolvimento de experiências que influenciem positivamente o desenvolvimento de destinos sustentáveis. Outro dos passos é a identificação dos fatores que influenciam/condicionam a experiência turística, de forma a entender como é que esta interage com estes fatores e vice-versa (Morgan \& Xu, 2010), de modo a tirar o melhor partido do desenvolvimento de ambientes próprios à criação de experiências turísticas, e consequentemente ao desenvolvimento turístico do destino.

Este artigo pretende analisar a produção científica presente na plataforma SCOPUS, desde 1970 até maio de 2017, relativamente à experiência turística e ao seu contributo para o desenvolvimento turísticos das áreas rurais. Para dar resposta a este objetivo, o presente artigo encontra-se dividido em quatro seç̧ões. A primeira corresponde à metodologia utilizada no presente artigo, onde se efetuou o levantamento dos estudos que foram desenvolvimentos no âmbito da economia de experiências, experiências turísticas e desenvolvimento turístico, nas áreas rurais. Já a segunda parte refere-se à identificação das dimensões da experiência turística tendo em consideração diferentes autores. $\mathrm{Na}$ seguinte parte, aborda-se o desenvolvimento dos destinos rurais e a sua relação com a experiência turística. Por fim, evidenciam-se as principais conclusões e pistas para investigações futuras.

\section{Metodologia}

Este artigo analisa artigos conceptuais e empíricos sobre a experiência turística e o desenvolvimento turístico, nas áreas rurais. Deste modo, o mesmo tem como objetivo identificar e analisar as diferentes dimensões da experiência turística, e posteriormente, perceber como esta pode contribuir para o desenvolvimento turístico das áreas rurais.

Para se atingir a primeira parte do objetivo proposto anteriormente, numa fase inicial, procedeu-se à realização de uma pesquisa na base de dados científica SCOPUS, durante o mês de maio de 2017, usando um conjunto de palavras-chaves 'experience economy', 'tourism', 'tourism experience' ou 'tourist experience' e 'rural'. Definiu-se que as palavras-chave poderiam aparecer no título, nas palavras-chave e resumo dos artigos, e limitou-se a pesquisa a artigos científicos. O período temporal considerado na presente pesquisa foi o permitido pela plataforma SCOPUS (1970-2017).

$\mathrm{Na}$ primeira fase da pesquisa obteve-se um resultado de 63 artigos que contemplam as palavras 'experience economy' e 'tourism', e destes, 13 focam-se no contexto rural ('experience economy'e 'tourism' e 'rural') (quadro 1). Para o presente artigo consideram-se os 63 artigos, de modo a entender não apenas como é operacionalizada a experiência em contexto rural, mas também a nível global. Numa fase posterior, considerou os 58 artigos que resultam da pesquisa 'tourism experience' ou 'tourist experience' e 'rural'. Neste contexto, salienta-se que existem três artigos que se repetem nas três pesquisas realizadas, nomeadamente os artigos associados aos autores Loureiro (2014), Quadri-felitti e Fiore (2013) e Quadri-felitti e Fiore (2016), e como tal apenas se considerou os presentes artigos uma vez.

Através do quadro 1, pode ainda verificar-se que a revista cientifica que tem um maior número de artigos publicados com as palavras 'experience economy' e 'tourism' é a revista Scandinavian Journal 
of Hospitality and Tourism. Já para as palavras 'experience economy', 'tourism' e 'rural' a revista é International Journal of Services and Standards. As principais autoras nas duas primeiras situações são a Fiore, A.M. e a Quadri-Felitti, D., associadas a universidades localizadas nos Estados Unidos. Contudo, quando se introduziram as palavras-chave 'tourism experience' ou 'tourist experience' e 'rural' já não se encontraram os mesmos resultados. A revista que se destaca com esta palavras-chave é o Journal of Sustainable Tourism e as principais autoras são Kastenholz, E. e Carneiro, M.J., que estão associadas à Universidade de Aveiro.

\section{Quadro 1: Análise quantitativa da conjugação das palavras economia de experiencias, turismo e rural}

\begin{tabular}{|c|c|c|c|}
\hline & $\begin{array}{l}\text { 'experience economy' e } \\
\text { 'tourism' }\end{array}$ & $\begin{array}{l}\text { 'experience economy' e } \\
\text { 'tourism' e 'Rural' }\end{array}$ & $\begin{array}{l}\text { 'tourism experience' ou } \\
\text { 'tourist experience' e } \\
\text { 'Rural' }\end{array}$ \\
\hline $\begin{array}{l}\text { Tipo de } \\
\text { análise }\end{array}$ & \multicolumn{3}{|l|}{ Análise quantitativa } \\
\hline Informação & \multicolumn{3}{|l|}{$\begin{array}{l}\text { - Título; } \\
\text { - Palavras-chave; } \\
\text { - Resumo }\end{array}$} \\
\hline $\begin{array}{l}\text { Período de } \\
\text { recolha de } \\
\text { dados }\end{array}$ & 2004-2017 & $2006-2016$ & $1999-2017$ \\
\hline $\begin{array}{l}\text { Número } \\
\text { de artigos } \\
\text { académicos }\end{array}$ & 63 & 13 & 58 \\
\hline $\begin{array}{l}\text { Principais } \\
\text { revistas } \\
\text { científicas }\end{array}$ & $\begin{array}{l}\text { - Scandinavian Journal of } \\
\text { Hospitality and Tourism } \\
\text { (n=6); } \\
\text { - Asia Pacific Journal of } \\
\text { Tourism Research }(\mathrm{n}=3) ; \\
\text { - International Journal } \\
\text { of Contemporary } \\
\text { Hospitality Management } \\
\text { (n=3); } \\
\text { - Journal of Hospitality } \\
\text { and Leisure Marketing } \\
\quad(\mathrm{n}=3) ; \\
\text { - Journal of Travel } \\
\text { Research }(\mathrm{n}=3) ; \\
\text { - Restantes revistas } \\
\text { científicas com pouca } \\
\text { representatividade }\end{array}$ & $\begin{array}{l}\text { - International Journal of } \\
\text { Services and Standards } \\
(\mathrm{n}=2) \\
\text { - Restantes revistas } \\
\text { científicas com pouca } \\
\text { representatividade }\end{array}$ & $\begin{array}{l}\text { - Journal of Sustainable } \\
\quad \text { Tourism }(\mathrm{n}=11) ; \\
\text { - Tourism Management } \\
\quad(\mathrm{n}=4) ; \\
\text { - Tourismos }(\mathrm{n}=3) ; \\
\text { - Worldwide Hospitality } \\
\quad \text { and Tourism Themes } \\
\quad(\mathrm{n}=3) ; \\
\text { - Restantes revistas } \\
\quad \text { científicas com pouca } \\
\text { representatividade }\end{array}$ \\
\hline $\begin{array}{l}\text { Autores com } \\
\text { mais artigos } \\
\text { publicados }\end{array}$ & $\begin{array}{l}\text { - Fiore, A.M. }(n=5) \\
\text { - Quadri-Felitti, D. }(n=3) \text {; } \\
\text { - Eide, D. }(n=2) ; \\
\text { - Oh, H. }(n=2) ; \\
\text { - Pearce, P.L. }(n=2)\end{array}$ & $\begin{array}{l}\text { - Fiore, A.M. }(n=4) \\
\text { - Quadri-Felitti, D. }(\mathrm{n}=3) \text {; }\end{array}$ & $\begin{array}{l}\text { - Kastenholz, E. }(\mathrm{n}=4) ; \\
\text { Carneiro, M.J. }(\mathrm{n}=3) ; \\
\text { - Eusébio, C. }(\mathrm{n}=2) ; \\
\text { - Ezeuduji, I.O. }(\mathrm{n}=2) ; \\
\text { - Fiore, A.M. }(\mathrm{n}=2) ; \\
\text { - Jepson, D. }(\mathrm{n}=2) ; \\
\text { - Lima, J. }(\mathrm{n}=2) ; \\
\text { - Nair, V. }(\mathrm{n}=2) ; \\
\text { - Sharpley, R. }(\mathrm{n}=2) ; \\
\text { - McIntosh, A.J. }(\mathrm{n}=2) ; \\
\text { - Quadri-Felitti, D. }(\mathrm{n}=2)\end{array}$ \\
\hline
\end{tabular}

Fonte: Elaboração Própria 
Com base no quadro 2, pode observar-se a evolução do número de artigos de acordo com as palavras-chave. Verificou-se que o número de artigos onde aparecem as palavras 'experience economy' e 'tourism', começou a aumentar a partir de 2013 e atingiu o seu auge em $2015(n=12)$. Pôde ainda observar-se que o número de artigos onde aparece as palavras 'experience economy' e 'tourism' e 'rural' é uma área recente de investigação, já que esta começou a ter maior representatividade a partir de 2012.

Relativamente ainda ao quadro 2 , pôde constatar-se que foram considerados relevantes para a análise das dimensões da experiência turística 15 artigos (apêndice A), lidos na íntegra. Para selecionar estes 15 artigos aplicou-se o seguinte processo: i) eliminar os artigos em que o título não se relacionava minimamente com o objetivo do estudo; ii) eliminar os artigos em que o resumo dos mesmos não se relacionava com o objetivo do estudo; iii) eliminar os artigos em que o resumo, os objetivos e a metodologia não se relacionavam com o objetivo do estudo.

\section{Quadro 2: Evolução do número de artigos na SCOPUS consoante os diferentes domínios, 1999 - 2017}

\begin{tabular}{|c|c|c|c|c|}
\hline Ano & $\begin{array}{c}\text { 'experience } \\
\text { economy' e 'tourism' }\end{array}$ & $\begin{array}{c}\text { 'experience } \\
\text { economy' e } \\
\text { 'tourism' e 'rural' }\end{array}$ & $\begin{array}{c}\text { "tourism experience" } \\
\text { ou "tourist } \\
\text { experience" e Rural }\end{array}$ & $\begin{array}{c}\text { Número de artigos } \\
\text { relevantes para } \\
\text { análise }\end{array}$ \\
\hline 1999 & - & - & 1 & - \\
\hline 2001 & - & - & 1 & - \\
\hline 2003 & - & - & 1 & - \\
\hline 2004 & 1 & - & - & - \\
\hline 2005 & - & - & 1 & - \\
\hline 2006 & 2 & 2 & 2 & - \\
\hline 2007 & 3 & 1 & 1 & 1 \\
\hline 2008 & 2 & - & 3 & - \\
\hline 2009 & 6 & - & 2 & 1 \\
\hline 2010 & 5 & - & 4 & 2 \\
\hline 2011 & 3 & 1 & 5 & 1 \\
\hline 2012 & 4 & 1 & 3 & 1 \\
\hline 2013 & 9 & 2 & 7 & 1 \\
\hline 2014 & 7 & 1 & 9 & 2 \\
\hline 2015 & 12 & 4 & 9 & 3 \\
\hline 2016 & 7 & 2 & 7 & 3 \\
\hline 2017 & 2 & - & 2 & - \\
\hline Total & 63 & 13 & 58 & 15 \\
\hline
\end{tabular}

Fonte: Elaboração Própria

Numa segunda fase, e para responder à segunda parte do objetivo, procedeu-se a uma nova pesquisa utilizando as palavras 'tourism experience' ou 'tourist experience', 'tourism development' ou 'tourist development' e 'rural'. Definiu-se igualmente que as palavras-chave poderiam aparecer no título, nas palavras-chave e resumo dos artigos, e limitou-se a pesquisa a artigos científicos. O período temporal foi o mesmo da pesquisa realizada anteriormente.

No quadro 3, pode verificar-se que o número de artigos publicados sobre a experiencia turística e o desenvolvimento turístico foi de 144, um valor relativamente elevado. Quando se restinguiu a pesquisa e se juntou todos os domínios (tourism experience' ou 'tourist experience' e 'tourism development' ou 'tourist development' e 'rural') que se pretende analisar no presente artigo, obteve-se um total de 19 artigos. Mais se acrescenta que os 19 artigos representam parte dos artigos obtidos a partir da pesquisa 
'tourism experience' ou 'tourist experience' e 'rural' ( $n=58)$, visto que as palavras-chave se repetem, porém na análise efetuada teve-se o cuidado de não considerar os mesmos artigos nas duas abordagens efetuadas e inclusive para cada abordagem selecionaram-se os artigos que efetivamente podiam dar uma melhor resposta a cada uma das partes do objetivo.

\section{Quadro 3: Análise quantitativa da conjugação das palavras experiência turística, desenvolvimento turístico e rural}

\begin{tabular}{|l|l|l|l|l|}
\hline $\begin{array}{l}\text { Tipo de } \\
\text { análise }\end{array}$ & Informação & $\begin{array}{c}\text { Período de } \\
\text { coleta de } \\
\text { dados }\end{array}$ & \multicolumn{1}{|c|}{ Palavras-chave de pesquisa } & $\begin{array}{c}\text { Número } \\
\text { de artigos } \\
\text { académicos }\end{array}$ \\
\hline $\begin{array}{l}\text { Análise } \\
\text { quantitativa }\end{array}$ & \begin{tabular}{l} 
- $\begin{array}{l}\text { - Paltulo; } \\
\text {-chave; } \\
\text { - Resumo }\end{array}$ \\
\cline { 2 - 4 }
\end{tabular} & $1982-2017$ & $\begin{array}{l}\text { 'tourism experience' ou 'tourist } \\
\text { experience' e 'tourism development' ou } \\
\text { 'tourist development' }\end{array}$ & 144 \\
\hline
\end{tabular}

Fonte: Elaboração Própria

O principal autor, para a primeira pesquisa, é o Richie, J.R.B. $(n=3)$, seguido por um conjunto de autores que publicaram dois artigos. As revistas que publicam um maior número de artigos são: Tourism Management ( $n=17)$, Annals of Tourism Research ( $n=13)$ e Current Issues in Tourism $(n=9)$. Quando se analisaram os resultados da pesquisa com o acrescimento da palavra 'rural' verificou-se que o principal autor já não é o mesmo, sendo que esta posição passa a ser representada por Ezeuduji, I.O. ( $n=2)$. A principal revista associada a esta temática é Journal of Sustainable Tourism $(n=5)$.

Por último, relativamente à metodologia, através da figura 1, pode observar-se a evolução do número de artigos com as palavras-chave 'tourism experience' ou 'tourist experience', 'tourism development' ou 'tourist development', e "rural". Verificou-se claramente que a presente área de investigação ('tourism experience' ou 'tourist experience', 'tourism development' ou 'tourist development') começou a ser mais estudada a partir de 2009, atingindo o seu auge em $2014(n=19)$. Quando se aplicou este domínio de investigação às áreas rurais (adição da palavra 'rural') constatou-se que a mesma é uma área muito recente de investigação, sendo que os anos com um maior número de artigos publicados foram os de $2014(n=3)$ e $2015(n=4)$.

\section{Figura 1: Evolução do número de artigos na SCOPUS consoante os diferentes domínios, 1982 - 2017}

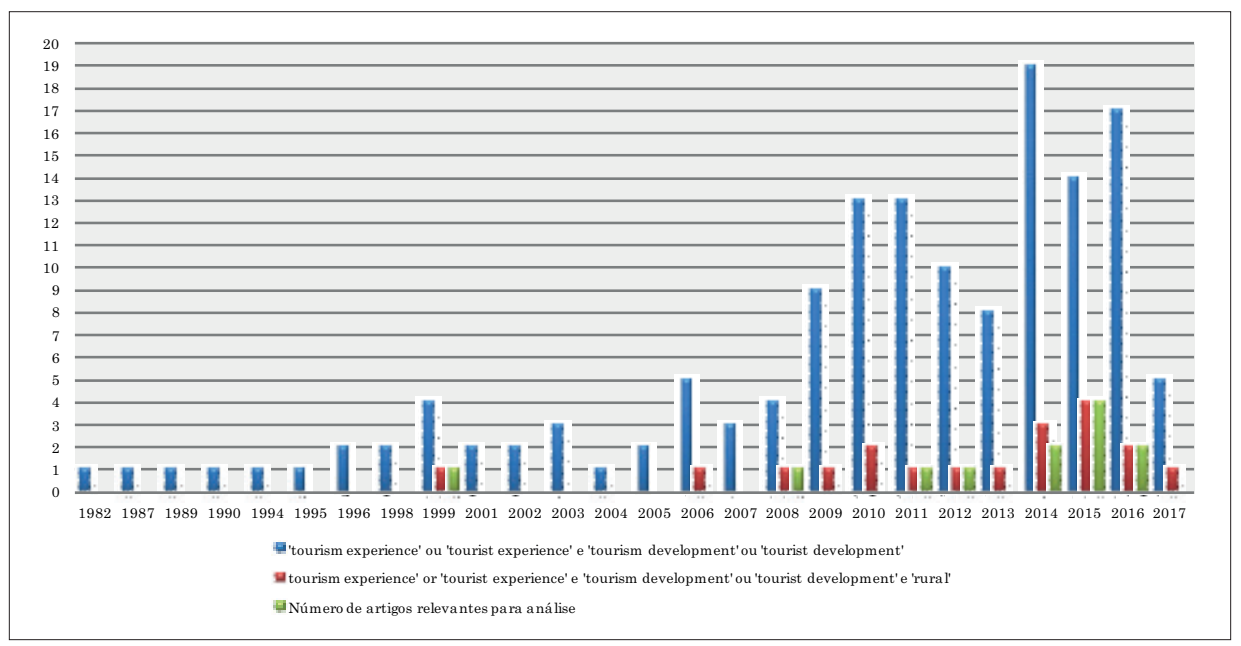

Fonte: Elaboração Própria 
Considerando que se pretende analisar o papel que as experiências turísticas podem ter no desenvolvimento turístico das áreas rurais, apenas se considerou na presente análise os artigos que se encontram inseridos no contexto rural. Assim, o número de artigos considerados para analisar esta temática foi de 12 (apêndice B). Mais se acrescenta que os critérios de seleção dos respetivos artigos foram os mesmos da pesquisa anterior.

\section{As dimensões da experiência turística}

A experiência turística é uma temática relativamente recente e que tem sofrido algumas alterações conceptuais que foram influenciadas pela evolução dos mercados e do comportamento dos consumidores, bem como pela investigação académica que tem vindo a ser desenvolvida. Isto faz com que a definição de experiência turística não seja consensual entre os diversos autores que investigam este tema, o que leva a diferentes operacionalizações do conceito.

O conceito de experiência foi mencionado pela primeira vez por Toffler (1970) (Knutson \& Beck, 2008) e mais tarde foi referido pelos autores Holbrook e Hirschman (1982). No entanto, só em 1998, o mesmo ganhou notoriedade através da abordagem realizada pelos autores Pine e Gilmore. Estes autores consideram que a experiência está dividida em quatro grandes dimensões - entretenimento, educativa, estética e fuga - que se encontram relacionadas com duas grandes divisões - a participação (ativa/passiva) e a relação com o meio ambiente (imersão/ absorção). Os referidos autores evidenciam que este modelo pode ser utilizado para analisar a experiência em qualquer contexto. Desde então, este modelo tem sido utilizado em diferentes domínios, incluindo a área do Turismo.

Repare-se que a crescente utilização deste modelo aplicado à área do Turismo é percetível através de uma simples e rápida análise dos estudos que resultaram da pesquisa efetuada na SCOPUS, onde vários são os autores que incorporaram este modelo nas suas investigações, mantendo-o inalterável ou com a adicionação de novas dimensões e/ou com a alteração dos itens que medem a experiência (Boo \& Lu, 2015; Hosany \& Witham, 2009; Loureiro, 2014; Mehmetoglu \& Engen, 2011; Oh, Fiore \& Jeoung, 2007; Quadri-felitti \& Fiore, 2013; Quadri-felitti \& Fiore, 2016; Song, Ahn \& Lee, 2015; Suntikul \& Jachna, 2016).

Contudo, antes da abordagem de Pine e Gilmore (1998) sobre a experiência, já outros autores tinham quantificado a experiência no âmbito do Turismo. Destacam-se os autores Otto e Ritchie (1996) que consideram que a experiência turística se encontra dividida em quatro dimensões - hedonics, peace of mind, involvement e recogition -, e o autor Ryan (1997, citado por Lee \& Smith, 2015) que divide a mesma em individual, entertainment, learning, or both.

Após a abordagem de Pine e Gilmore (1998), somaram-se muitas outras abordagens que tentaram conceptualizar/operacionalizar a experiencia turística. Aho (2001) definiu que a experiência turística se encontra dividida em emotional experiences, learning experiences, practical experiences e transformational experiences. Para os autores Dube e Le Bel (2003, citado por Scott, Laws \& Boksberger, 2009) a experiência possui quatro dimensões, nomeadamente sensory (or physical), social, emotional, e intellectual. Segundo Quan e Wang (2004), a experiência turística está dividida em peak touristic experience e supporting consumer experience. Já os autores Tarssanem e Kylänen (2005, citado por Mehmetoglu \& Engen, 2011) consideram que a experiência pode ser dividida em sete dimensões (individuality, authenticity, story, multi-sensory perception, contast e interaction) representadas num triângulo pela presente ordem, a esta abordagem os autores deram o nome de "the experience pyramid". Em 2007, os autores Oh et al., aplicaram a escala de Pine e Gilmore (1998) à área do Turismo, e dois anos mais tarde, os autores Hosany e Witham (2009) utilizaram a referida escala no seu estudo.

Em 2011, Tung e Ritchie consideram que as quatro dimensões que melhor representam a experiência turística são affect, expectations, consequentiality e recollection. No mesmo ano, Walls et al. (2011) definiram que a experiência turística se encontra dividida em quatro dimensões - ordinary, extraordinary, cognitive e emotive. Já Ballantyne, Packer e Sutherland (2011) consideraram que a experiência se divide em sensory impressions, emotional affity, reflective response e behaviour response. Um ano mais tarde, Kim, Ritchie e McCormick (2012), adotaram uma outra perspetiva, e defenderam que a experiência turística está dividida em diversas dimensões, sendo estas hedonism, involvement, local culture, refreshment, meaningfulness, knowledge e novelty. Após diferentes abordagens, e com base num conjunto de publicações de outros autores, Kastenholz, Carneiro, Marques e Lima (2012) salientaram no seu estudo "Understanding and managing the rural tourism experience - The case of historical village in Portugal" que geralmente a experiência turística está dividida em quatro dimensões, tais como emotional, social, cognitive e sensescape. 
Mediante o exposto, pôde constatar-se que a experiência turística envolve diferentes dimensões, e que existem diferentes modelos e abordagens que permitem operacionalizar a mesma. Contudo, apesar das diferentes abordagens mencionadas anteriormente, e tendo por base a pesquisa efetuada na plataforma SCOPUS com as palavras-chave 'experience economy' e 'tourism'e 'tourism experience' ou 'tourist experience' e 'rural', pôde verificar-se que em grande parte dos estudos predomina a utilização do modelo criado por Pine e Gilmore (1998) ou do modelo de Pine e Gilmore adaptado ao Turismo que foi desenvolvido por Oh et al. (2007) (quadro 4). Através do quadro 4, e no que concerne ao contexto da experiência, pode constatar-se que cerca de $1 / 3$ dos estudos selecionados se desenvolveram em contexto rural.

\section{Quadro 4: As dimensões da experiência turística}

\begin{tabular}{|c|c|c|}
\hline Autor & Contexto & Dimensões da experiência \\
\hline Oh et al.(2007) & $\begin{array}{l}\text { Indústria 'Bed and } \\
\text { Breakfast' }\end{array}$ & $\begin{array}{l}\text { Education; Esthetics; } \\
\text { Entertainment; Escapism }\end{array}$ \\
\hline Hosany e Witham (2009) & $\begin{array}{l}\text { Indústria de } \\
\text { Cruzeiros }\end{array}$ & $\begin{array}{l}\text { Education; Esthetics; } \\
\text { Entertainment; Escapism }\end{array}$ \\
\hline Xu e Chan (2010) & $\begin{array}{l}\text { Circuitos } \\
\text { Organizados (Pacotes } \\
\text { turísticos) }\end{array}$ & $\begin{array}{l}\text { Recognition and Escapism; } \\
\text { Peace of Mind and Relaxing; } \\
\text { Hedonics; e Involvement }\end{array}$ \\
\hline Mehmetoglu e Engen (2011) & Festival e museu & $\begin{array}{l}\text { Education; Esthetics; } \\
\text { Entertainment; Escapism }\end{array}$ \\
\hline Kastenholz et al. (2012) & Destino Rural & $\begin{array}{l}\text { Afective; Sensory; Cognitive; } \\
\text { Behaviour }\end{array}$ \\
\hline Quadri-felitti e Fiore (2013) & Região vinícola & $\begin{array}{l}\text { Education; Esthetics; } \\
\text { Entertainment; Escapism }\end{array}$ \\
\hline Loureiro (2014) & Destino Rural & $\begin{array}{l}\text { Education; Esthetics; } \\
\text { Entertainment; Escapism }\end{array}$ \\
\hline Agapito, Valle e Mendes (2014) & Destino Rural & Sensory \\
\hline Song et al. (2015) & Megaeventos & $\begin{array}{l}\text { Education; Esthetics; } \\
\text { Entertainment; Escapism }\end{array}$ \\
\hline Lee e Smith (2015) & $\begin{array}{l}\text { Sítios históricos e } \\
\text { museus }\end{array}$ & $\begin{array}{l}\text { Escapism; Entertainment; } \\
\text { Education; Culture identity; } \\
\text { Relation develop }\end{array}$ \\
\hline Boo e Lu (2015) & Eventos & $\begin{array}{l}\text { Education; Esthetics; } \\
\text { Entertainment; Escapism }\end{array}$ \\
\hline Geus, Richards e Toepoel (2016) & Eventos & $\begin{array}{l}\text { Affective engagement; } \\
\text { cognitive engagement; } \\
\text { physical engagement; } \\
\text { experiencing novelty }\end{array}$ \\
\hline Quadri-felitti e Fiore (2016) & Região vinícola & $\begin{array}{l}\text { Education; Esthetics; } \\
\text { Entertainment; Escapism }\end{array}$ \\
\hline Suntikul e Jachna (2016) & $\begin{array}{l}\text { Centro Histórico de } \\
\text { Macau }\end{array}$ & $\begin{array}{l}\text { Education; Esthetics; } \\
\text { Entertainment; Escapism }\end{array}$ \\
\hline
\end{tabular}

Fonte: Elaboração Própria

No quadro 4, pode igualmente verificar-se que 14 dos 15 artigos analisados são empíricos. Os estudos que analisam a experiência turística utilizam diferentes metodologias, porém predominam as de cariz quantitativo, com recurso à aplicação de inquéritos por questionário (quadro 5). A vertente mais qualitativa deste tipo de estudos é apenas aplicada por dois autores, que recorrem às entrevistas para recolherem dados primários (quadro 5). 


\section{Quadro 5: Técnicas de recolha dos dados e análise dos resultados, considerandos os diferentes autores}

\begin{tabular}{|l|l|l|}
\hline \multicolumn{1}{|c|}{$\begin{array}{c}\text { Análise dos } \\
\text { dados }\end{array}$} & $\begin{array}{l}\text { Instrumento (s) de } \\
\text { recolha de dados }\end{array}$ & \multicolumn{1}{c|}{ Autores } \\
\hline Quantitativos & Questionário & $\begin{array}{l}\text { Agapito et al. (2014) Hosany e Witham (2009); Lee e Smith } \\
\text { (2015); Loureiro (2014); Mehmetoglu e Engen (2011); Quadri- } \\
\text {-felitti e Fiore (2013); Quadri-felitti e Fiore (2016); Song et } \\
\text { al. (2015); Suntikul e Jachna (2016);Xu e Chan (2010) }\end{array}$ \\
\hline Qualitativos & Entrevistas & Kastenholz et al. (2012) \\
\hline \multirow{2}{*}{ Mistos } & $\begin{array}{l}\text { Questionários; } \\
\text { Entrevistas }\end{array}$ & Oh et al. (2007) \\
\cline { 2 - 4 } & $\begin{array}{l}\text { Questionários; } \\
\text { Focus groups }\end{array}$ & Geus et al. (2016) \\
\hline
\end{tabular}

\section{Fonte: Elaboração Própria}

Pode ainda observar-se, através do quadro 6, que a maioria dos estudos analisa a experiência turística apenas sob o ponto de vista da procura (85\%) (Agapito et al., 2014; Boo \& Lu, 2015; Hosany \& Witham, 2009; Lee \& Smith, 2015; Loureiro, 2014; Mehmetoglu \& Engen, 2011; Quadri-felitti \& Fiore, 2013; Song et al., 2015; Suntikul \& Jachna, 2016; Xu \& Chan, 2010)a rich body of research on applications of the experience economy concepts has appeared in the marketing literature. However, academic investigations on the measurement of tourism experiences are very recent. Drawing on Oh, Fiore and Jeoung (2007, existindo portanto apenas três estudos que consideram a oferta e a procura (Kastenholz et al., 2012; Oh et al., 2007; Quadri-felitti \& Fiore, 2016). Mais se acrescenta que se considera o estudo dos autores Kastenholz et al. (2012) o mais completo em termos de análise sob diferentes pontos de vista, já que para além de considerar a oferta e a procura, considera ainda diferentes elementos da oferta - os residentes e as entidades públicas.

\section{Quadro 6: Análise da experiência turística sobre diferentes perspetivas}

\begin{tabular}{|l|c|c|c|c|}
\hline \multirow{2}{*}{ Autores } & \multirow{2}{*}{ Procura } & Residentes & $\begin{array}{c}\text { Entidades } \\
\text { públicas }\end{array}$ & $\begin{array}{c}\text { Agentes do sector } \\
\text { privado }\end{array}$ \\
\cline { 4 - 5 } & & $\mathrm{x}$ & & \\
\hline Oh et al. (2007) & $\mathrm{x}$ & & & $\mathrm{x}$ \\
\hline Hosany e Witham (2009) & $\mathrm{x}$ & & & $\mathrm{X}$ \\
\hline Xu e Chan (2010) & $\mathrm{x}$ & & & \\
\hline Mehmetoglu e Engen (2011) & $\mathrm{x}$ & $\mathrm{x}$ & & \\
\hline Kastenholz et al. (2012) & $\mathrm{x}$ & & & \\
\hline Quadri-felitti e Fiore (2013) & $\mathrm{x}$ & & & \\
\hline Loureiro (2014) & $\mathrm{x}$ & & & \\
\hline Agapito et al. (2014) & $\mathrm{x}$ & & & \\
\hline Song et al. (2015) & $\mathrm{x}$ & & & \\
\hline Lee e Smith (2015) & $\mathrm{x}$ & & & \\
\hline Boo e Lu (2015) & $\mathrm{x}$ & & & \\
\hline Quadri-felitti e Fiore (2016) & & & & \\
\hline Suntikul e Jachna (2016) & & & \\
\hline
\end{tabular}

Fonte: Elaboração Própria 
Apesar de, como já mencionado anteriormente, grande parte dos estudos analisarem a experiência turística com base no modelo de Pine e Gilmore (1998), os autores tinham objetivos diferentes e iam além da simples mensuração da experiência (quadro 7). Os autores Oh et al. (2007) e Hosany e Witham (2009) pretendiam analisar a experiência turística e as consequências da mesma. Oh et al. (2007) e Hosany e Witham (2009) concluíram que todas as dimensões estavam correlacionadas com as consequências da viagem, sendo que no estudo de $\mathrm{Oh}$ et al. (2007) as dimensões educação e estética estavam relacionadas com a consequência Arousal. Importa salientar que a dimensão estética teve uma relação significada com todas as consequências da viagem (arousal, memory, overall experience e guest satisfatction). Em contrapartida, as dimensões fuga e entretenimento aparecem como sendo pouco significativas nas consequências da viagem, ao contrário do que é relatado na literatura. No mesmo seguimento de ideias, os autores Hosany e Witham (2009) também concluíram que a dimensão estética foi a que teve uma relação mais significativa com as consequências da viagem (arousal, memory, overall perceived quality, satisfaction, e intention to recommend), seguida da dimensão entretenimento. Contrariamente, as dimensões educação e fuga foram consideradas as menos importantes nas consequências da viagem.

Sob o mesmo ponto de vista, mas considerando apenas a satisfação, os autores Mehmetoglu e Engen (2011) inquiriram 192 visitantes em dois contextos diferentes e concluíram que as dimensões da educação e do entretenimento não afetavam o nível de satisfação dos visitante num dos contexto (museu), já no outro contexto essas dimensões passaram a dizer respeito à fuga e ao entretenimento (festival). Isto permite concluir que é necessário criar ambientes propícios à criação de diferentes tipos de experiências, dependendo do contexto que se pretende explorar. Song et al. (2015) chegaram a uma conclusão semelhante à dos autores Hosany e Witham (2009), visto que as dimensões estética e entretenimento foram as que mais influenciaram a satisfação dos visitantes, e que as dimensões fuga e educação foram as que menos influenciaram a satisfação. Os presentes autores evidenciam que efetivamente a dimensão estética foi a que mais influenciou positivamente a satisfação. Ainda relativamente a esta tématica, os autores Quadri-felitti e Fiore (2013) inquiriam 970 turistas e concluiram que as dimensões estética e educação influenciavam positivamente a satisfação dos turistas. Isto permite afirmar que a importância que as diferentes dimensões possuem nas consequências da viagem vai depender do próprio contexto/ ambiente da experiência.

Outra das relações a comparar por diferentes autores é a relação entre a experiência turística e a memória. Quadri-felitti e Fiore (2013) constataram que apenas duas dimensões - estética e educação - influenciavam significativamente a memoria, sendo que a estética sobressaiu-se claramente comparativa com a educação. Os autores referem que a experiencia estética deve ser considerada uma atração central no Turismo Rural. No estudo desenvolvido por Loureiro (2014) a dimensão estética da experiência é a mais relevante na formação da experiência global em contexto rural, seguida pelas dimensões fuga e entretenimento. Já no estudo de Boo e Lu (2015) a dimensão mais relevante na formação da experiência é o entretenimento, seguida pela estética.

Quadro 7: Variáveis dependentes

\begin{tabular}{|c|c|c|c|c|c|c|c|}
\hline $\begin{array}{c}\text { Variáveis } \\
\text { dependentes }\end{array}$ & Arousal & Memory & Quality & Satisfaction & $\begin{array}{l}\text { Behaviour } \\
\text { intention }\end{array}$ & $\begin{array}{c}\text { Positive } \\
\text { affect }\end{array}$ & $\begin{array}{c}\text { Telepresence } \\
\text { and time } \\
\text { distortion }\end{array}$ \\
\hline Oh et al. (2007) & $\mathrm{X}$ & $\mathrm{x}$ & $\mathrm{x}$ & $\mathrm{x}$ & & & \\
\hline $\begin{array}{l}\text { Hosany e Witham } \\
(2009)\end{array}$ & $\mathrm{X}$ & $\mathrm{x}$ & $\mathrm{x}$ & $\mathrm{x}$ & $\mathrm{x}$ & & \\
\hline Xu e Chan (2010) & & & & $\mathrm{x}$ & $\mathrm{x}$ & & \\
\hline $\begin{array}{l}\text { Mehmetoglu e } \\
\text { Engen (2011) }\end{array}$ & & & & $\mathrm{x}$ & & & \\
\hline $\begin{array}{l}\text { Quadri-felitti e } \\
\text { Fiore (2013) }\end{array}$ & & $\mathrm{x}$ & & $\mathrm{x}$ & $\mathrm{x}$ & & \\
\hline Loureiro (2014) & $\mathrm{X}$ & $\mathrm{x}$ & & & & & \\
\hline $\begin{array}{l}\text { Agapito et al. } \\
\text { (2014) }\end{array}$ & & & & & & & \\
\hline Song et al. (2015) & & & & $\mathrm{x}$ & & & \\
\hline Boo e Lu (2015) & & $\mathrm{x}$ & & & & $\mathrm{x}$ & $\mathrm{X}$ \\
\hline
\end{tabular}

Fonte: Elaboração Própria 
Em género de sintese é claramente notório que existe uma tendência para analisar a influência da dimensão da experiência na satifação e memória. Através da exposição realizada anteriormente sobre as principais conclusões dos estudos, verifica-se que a dimensão estética da experiência é indispensável na formação global da experiência por parte dos visitantes, e que a mesma tem uma relação positiva com as consequências da viagem.

\section{A experiência turística e o desenvolvimento do turismo nas áreas rurais}

Aquando da análise dos 12 artigos (10 artigos empíricos e 2 conceptuais) resultantes da pesquisa 'tourism experience' ou 'tourist experience', 'tourism development' ou 'tourist development', e 'rural', constatou-se que os estudos estavam divididos em três grandes áreas, que se encontravam interligadas entre si (quadro 8). Por conseguinte, existem artigos que abordam o desenvolvimento do turismo nas áreas rurais de forma abrangente (Dissart \& Marcouiller, 2012; Ezeuduji, 2015; Lun, Pechlaner \& Volgger, 2016; Murphy \& Williams, 1999), e outros que apresentam abordagens mais específicas, nomeadamente o estudo de Tolstad (2014) que analisa o papel das redes no turismo em áreas rurais; o estudo de Kim e Jamal (2015) que analisa especificamente a importância da agricultura nas áreas rurais, e o estudo de Idziak, Majewski e Zmyslony (2015) que analisa o papel do envolvimento da comunidade local no desenvolvimento de uma aldeia. Outros estudos analisam as perceções e atitudes dos residentes, agentes da oferta, entidade públicas, e visitantes face ao desenvolvimento do turismo em áreas rurais (quadro 8). Finalmente, a terceira categoria refere-se aos estudos que analisam a paisagem nas áreas rurais (Carneiro, Lima \& Silva, 2015; Cebrián \& Sánchez, 2016). Neste contexto, visto que se pretende analisar o contributo da experiência turística no desenvolvimento do turismo em áreas rurais, apenas se vai analisar o que cada um dos mesmos refere sobre esta temática.

\section{Quadro 8: Categorização dos estudos analisados}

\begin{tabular}{|ll|}
\hline \multicolumn{1}{|c|}{ Tipos de estudos } & \multicolumn{1}{c|}{ Autores } \\
\hline $\begin{array}{l}\text { 1. Estudos que analisam relação entre o turismo e o } \\
\text { desenvolvimento das áreas rurais (em geral) }\end{array}$ & $\begin{array}{l}\text { Dissart e Marcouiller (2012); Ezeuduji (2015); } \\
\text { Lun et al. (2016); Murphy e Williams (1999) }\end{array}$ \\
$\begin{array}{l}\text { 1.1. Estudos que analisam particularmente o papel das } \\
\text { redes no turismo em áreas rurais }\end{array}$ & Tolstad (2014) \\
$\begin{array}{l}\text { 1.2. Estudos que analisam especificamente a } \\
\text { agricultura em áreas rurais }\end{array}$ & Kim e Jamal (2015) \\
$\begin{array}{l}\text { 1.3. Estudos que analisam principalmente a } \\
\text { participação da comunidade local }\end{array}$ & Idziak et al. (2015) \\
\hline $\begin{array}{l}\text { 2. Estudos que analisam as perceções e atitudes dos } \\
\text { residentes, e/ou agentes privados da oferta, e/ou } \\
\text { entidades públicas, e/ou visitantes face ao turismo } \\
\text { em áreas rurais }\end{array}$ & $\begin{array}{l}\text { Eusébio, Kastenholz e Breda (2014); Ezeuduji e } \\
\text { Rid (2011); Lepp (2008) }\end{array}$ \\
\hline $\begin{array}{l}\text { 3. Estudos que analisam a paisagem em áreas rurais } \\
\text { Estudis }\end{array}$ & Carneiro et al. (2015); Cebrián e Sánchez (2016) \\
\hline
\end{tabular}

Fonte: Elaboração Própria

As áreas rurais estão associadas a valiosos recursos (Sharpley \& Stone, 2011) e determinadas atividades económicas (especialmente ligadas ao setor primário). Contudo, a importância do setor primário nestas áreas tem vindo a diminuir e a perder relevância económica (Tolstad, 2014), possibilitando assim o surgimento de outros tipos de atividades económicas que permitam beneficiar as áreas rurais, sendo de destacar o Turismo. Deste modo, o conhecimento e compreensão dos aspetos económicos, sociais, culturais e históricos destas áreas permitem o desenvolvimento sustentável do turismo. Através da leitura exaustiva dos 12 artigos verificou que são diversos os autores que referem várias vezes o termo 'desenvolvimento sustentável do turismo', porém é rara a sua quantificação ou mensuração. Apenas os autores Eusébio et al. (2014) referem que existem diferentes dimensões do desenvolvimento sustentável que foram evoluindo ao longo dos anos, sendo que inicialmente estas dimensões eram constituídas por 
três dimensões - económica, ambiental e sociocultural. Os autores referidos anteriormente evidenciaram a abordagem realizada por Choi e Sirakaya (2006, citado por Eusébio et al., 2014) que adicionou duas dimensões para além das três iniciais (tecnologia e política). Aplicando esta abordagem ao Turismo, e segundo Eusébio et al. (2014), o desenvolvimento sustentável do turismo deve satisfazer as necessidades e desejos de todos os stakeholders envolvidos e tanto quanto possível proteger os recursos que sustentam o turismo, sendo estes os recursos naturais e culturais.

Neste contexto, as áreas rurais são ricas em características naturais, culturais e sociais únicas, onde estas podem ser utilizadas no desenvolvimento de experiências mais autênticas e memoráveis (Ezeuduji, 2015; Lun et al., 2016; Murphy \& Williams, 1999). Murphy e Williams (1999) referem que o ponto-chave para o desenvolvimento de experiências autênticas passa pelo aproveitamento das oportunidades que estas áreas oferecem, como o contato com as características naturais e culturais do destino, as paisagens, e o contacto com a comunidade local. Ezeuduji (2015) considera que os recursos permitem, cada vez mais, aos destinos rurais ganharam vantagem competitiva. Contudo, Murphy e Williams (1999) alertam para o facto de estas oportunidades terem de ser cuidadosamente planeadas para que não haja um choque cultural e/ou incapacidade de transmitir a autenticidade destes locais.

Para além da utilização responsável e estratégica dos recursos endógenos do destino, Lun et al. (2016) consideram ainda que um dos fatores de sucesso para o desenvolvimento dos destinos rurais assenta nas características dos serviços. Estes devem ser únicos, de elevada qualidade e que proporcionem experiências. Mais se acrescenta que estes autores também salientam a ideia de que a criação de bens e serviços de elevada qualidade é um critério crucial para o sucesso do desenvolvimento do turismo nas áreas rurais, sendo que estes produtos devem ser desenvolvidos considerando os recursos naturais e $o$ património cultural existentes numa dada região.

Em suma, a criação de um ambiente propício ao desenvolvimento de experiências turísticas deverá incorporar os recursos endógenos da região (Ezeuduji, 2015; Lun et al., 2016; Murphy \& Williams, 1999), de modo a promover e a potenciar estes mesmos recursos, e a atrair os segmentos de mercado que mais os valorizam. Isto possibilitará, por um lado, o reconhecimento dos recursos, e por outro lado, o desenvolvimento de experiências que estimularam o crescimento do Turismo numa dada região (Lun et al., 2016). Consequentemente, o desenvolvimento do Turismo contribui para o desenvolvimento sustentável das áreas rurais (Eusébio et al., 2014).

Repare-se que através da análise efetuada aos 12 artigos pôde confirmar-se que é difícil entender de forma objetiva e quantitativa o poder que a experiência turística possui no desenvolvimento do turismo e das áreas rurais. Contudo, como já mencionado no capítulo anterior, as experiências representam uma nova etapa na economia, e isto é bastante visível na área do Turismo. Assim, observou-se que uma parte dos estudos referiu a importância que os recursos endógenos têm nas experiências turísticas (como visto anteriormente), e outros focaram no estudo de produtos e atividades económicas como forma de incorporação do Turismo na economia do local.

Neste último cenário, as experiências turísticas, em áreas rurais, podem estar assentes nos produtos locais (Eusébio et al., 2014; Kim \& Jamal, 2015; Tolstad, 2014) e na confeção dos mesmos. Isto permite beneficiar o desenvolvimento turístico e o local (Tolstad, 2014), uma vez que permite que a agricultura continue a ser uma atividade económica importante nestas regiões (Ezeuduji \& Rid, 2011), e valoriza o conhecimento social, cultural e histórico da comunidade local (Kim \& Jamal, 2015). No que concerne, ainda, à agricultura e às quintas associadas às áreas rurais (Idziak et al., 2015), outro tipo de experiências pode ser desenvolvido para acrescentar uma componente educacional (Kim \& Jamal, 2015). Assim, podem ser desenvolvidas experiências que privilegiem o trabalho na quinta/floresta, o contato com os animais, a ajuda na jardinagem e a realização de arranjos florais (Idziak et al., 2015). Isto permite o desenvolvimento do turismo, bem como o desenvolvimento sustentável das áreas rurais, já que utiliza de forma sustentável a natureza, permite a transmissão de culturas e saberes entre a comunidade local e o visitante, e estimula a economia local. Já o desenvolvimento de experiências culturais pode motivar as comunidades locais a preservarem a sua herança cultural (Ezeuduji \& Rid, 2011), bem como a fazerem com que a sua cultura seja melhor percebida e entendida pelos visitantes (Lepp, 2008).

O desenvolvimento de experiências em contextos esteticamente marcantes é também um fator a considerar, visto que de acordo com Carneiro, Lima e Silva (2017) a paisagem natural tem um contributo importante na satisfação dos visitantes, e segundo Murphy e Williams (1999) representa um dos fatores motivacionais dos turistas que procuram estas áreas. Repara-se que esta paisagem é influenciada pela presença de campos cultivados pelo Homem (Carneiro et al., 2017), refletindo mais uma vez a importância das características naturais, culturais e sociais (Cebrián \& Sánchez, 2016). 
Mediante o exposto, pôde constatar-se que o desenvolvimento de ambientes propícios à criação de experiências turísticas incorpora diferentes tipos de recursos, que se bem aproveitados em diferentes dimensões, podem desenvolver o Turismo num dado destino, e consequentemente desenvolver de forma sustentável a região.

Ao se analisar, em particular, os 10 artigos empíricos, verificou-se que em 9 dos 10 estudos empíricos predomina uma abordagem qualitativa, com recurso, maioritariamente à aplicação de entrevistas (quadro 9). Para além das entrevistas, existem autores que complementaram os seus estudos com outros instrumentos de recolha de dados, como a observação participante, o focus group e as entrevistas informais. Os autores Cebrián e Sánchez (2016) utilizaram a vertente quantitativa, através da aplicação de questionários. Repara-se que Murphy e Williams (1999) foram os únicos autores (considerando os 10 artigos analizados) que utilizaram dados primários e secundários no seu estudo.

\section{Quadro 9: Técnicas de recolha de dados e análise dos resultados, considerando os diferentes autores}

\begin{tabular}{|c|c|c|c|}
\hline $\begin{array}{l}\text { Tipo de } \\
\text { dados }\end{array}$ & Análise dos dados & $\begin{array}{l}\text { Instrumento (s) de } \\
\text { recolha de dados }\end{array}$ & Autores \\
\hline \multirow{5}{*}{$\begin{array}{l}\text { Dados } \\
\text { primários }\end{array}$} & Quantitativos & Questionário & Cebrián e Sánchez (2016) \\
\hline & \multirow{4}{*}{$\begin{array}{l}\text { Qualitativos } \\
\text { Observação participante } \\
\text { Entrevistas informais } \\
\text { Focus group }\end{array}$} & Entrevistas & 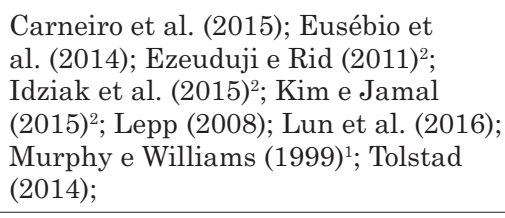 \\
\hline & & Kim e Jamal $(2015)^{2}$ & \\
\hline & & Idziak et al. $(2015)^{2}$ & \\
\hline & & Ezeuduji e Rid $(2011)^{2}$ & \\
\hline $\begin{array}{l}\text { Dados } \\
\text { secundários }\end{array}$ & & & Murphy e Williams (1999) ${ }^{1}$ \\
\hline
\end{tabular}

Fonte: Elaboração Própria

Neste cenário, constatou-se que os estudos que de alguma forma analisam o desenvolvimento turístico nas áreas rurais são predominantemente qualitativos, contrariamente aos estudos que analisam a experiência turística que são maioritariamente quantitativos. Tal aspeto pode estar relacionado com o facto de a temática 'experiências turísticas' ser um tema menos recente quando comparado com os estudos relacionados com o desenvolvimento e a experiência turística em contexto rural, necessitando-se assim de uma abordagem qualitativa para explorar o tema em questão.

Adicionalmente, e considerado a multiplicidade de lugares que podem ser inseridos nas áreas rurais, constatou-se que os estudos analisados $(n=10)$ são aplicados em diferentes contextos. Metade dos estudos $(n=5)$ foram realizados em aldeias, dois em áreas montanhosas, um num subcontinente, um num vale, e um considerou o país.

Tendo como referência os 10 artigos empíricos analisados em profundidade, observou-se seis dos mesmos desenvolveram os seus estudos sobre o ponto de vista da oferta, e apenas um dos estudos se dedicou à análise de duas perspetivas - oferta e procura. Sobre o estudo das duas perspetivas, merece destaque a investigação de Eusébio et al. (2014), que se dedica à análise das perceções dos vários stakeholders - procura (visitantes) e oferta (residentes, agentes da oferta do setor privado e entidades responsáveis pelo planeamento do turismo) sobre a atividade turística e suas implicações no desenvolvimento sustentável de uma aldeia, localizada em Portugal. 
Em suma, a revisão dos 12 artigos permitiu constatar que o desenvolvimento de experiências turísticas em áreas rurais e o seu impacte no desenvolvimento turístico/sustentável do destino é abordado de diferentes formas de acordo com diferentes autores e âmbitos dos estudos. Assim, não existem indicadores claros que permitam mensurar a importância da criação de experiências para o desenvolvimento sustentável do destino. No entanto, admite-se que como as experiências turísticas são um tema recente e difícil de mensurar faz com que a criação de indicadores de mensurabilidade do contributo da experiência para o desenvolvimento sustentável do destino não seja, para já, uma tarefa simples.

\section{Conclusões e futuras investigações}

O presente artigo teve como objetivo fazer uma reflecção tendo como base a revisão da literatura efetuada sobre as dimensões da experiência turística, e consequente contributo da mesma para o desenvolvimento do turismo nas áreas rurais. $\mathrm{O}$ crescente reconhecimento da importância do desenvolvimento do turismo em áreas rurais é percetível através da análise dos estudos publicados neste domínio, já que nos últimos três anos foram produzidos mais de metade dos trabalhos publicados desde 1999. Este reconhecimento resultou das transformações que ocorrem nas tendências de mercado e nas alterações do comportamento dos consumidores.

Desta forma é fundamental entender como é que o turismo pode ajudar a desenvolver estes territórios, não apenas a nível económico, mas também aos níveis social, cultural e ambiental. Uma pesquisa efetuada sobre a relação entre a experiência turística e o desenvolvimento do turismo em áreas rurais permitiu concluir que, a criação de ambientes propícios ao desenvolvimento de experiências que valorizam as características e recursos únicos destas áreas pode ser uma forma de desenvolver sustentavelmente o Turismo. Isto porque os visitantes atuais procuram usufruir de experiências autênticas, mais individualizadas, diversificadas e de elevada qualidade (Carvão, 2009; Lun et al., 2016; Swarbrooke \& Horner, 2002).

No século passado, iniciou-se o estudo da experiência turística, contudo esta ainda é considerada um tema recente de investigação, visto que continua a ser difícil de definir e operacionalizar este tipo de experiência. Isto é o resultado da experiência ser um fenómeno bastante complexo, que pode agregar diferentes dimensões, sendo que as mesmas podem ter um caráter mais ou menos subjetivo/objetivo. Acresce-se a esta complexidade o facto da experiência turística possuir uma conotação pessoal e de variar de acordo com o contexto em que o visitante se encontra (Andersson, 2007; Huijibens, 2012; Kim et al., 2012; Pine \& Gilmore, 1998; Schmitt, 1999; Sharpley \& Stone, 2011; Tung \& Ritchie, 2011)which are indeed memorable, directly determine a business $\backslash$ u2019s ability to generate revenue (Pine and Gilmore 1999. Considerando os diversos estudos analisados sobre a experiência turística, verificou-se um consenso no que respeita à importância de se continuarem a desenvolver estudos que analisem a mesma. Alguns autores referem que continua a existir a necessidade de melhorar as escalas que medem e analisam este tipo de experiências (Hosany \& Gilbert, 2010; Kim et al., 2012; Oh et al., 2007). Ainda relativamente às dimensões da experiência, constatou-se que a dimensão estética tem uma relação positiva bastante significativa com as consequências da viagem. Assim, e dado o número reduzido de estudos que analisa a dimensão sensorial da experiência (que engloba a dimensão estética) considera-se relevante o desenvolvimento de estudos que abordem esta temática.

Outra conclusão que se pôde retirar da análise efetuada foi que a maioria dos estudos que analisam as experiências turísticas centram-se numa análise quantitativa sob o ponto de vista da procura. Já quando se passa para a análise de como a experiência turística pode influenciar o desenvolvimento do turismo no destino, verifica-se o oposto. Assim, acredita-se que é fundamental desenvolver mais estudos que adotem um abordagem mista e que contemplem não apenas a procura, mas outros agentes que estão inseridos no setor do Turismo (exemplo: agentes da oferta, residentes, entidades públicas), pois isto permitirá entender melhor o problema que se está a analisar.

Considerando os artigos que abrangem a temática do desenvolvimento nas áreas rurais, verificou-se que existe um elevado número de artigos que fala sobre o desenvolvimento turístico sustentável, no entanto, existem poucos que analisam em profundidade as várias dimensões do desenvolvimento turístico sustentável. Desta forma, considera-se relevante a realização de estudos que possam melhor identificar e criar itens de mensuração das dimensões do desenvolvimento turístico sustentável em áreas rurais.

Lepp (2008) refere que a comunidade local deve ter algum conhecimento básico do Turismo, numa fase inicial de desenvolvimento, de modo a poder atenuar parcialmente algumas atitudes menos positivas relativamente ao Turismo e ao visitante. $\mathrm{O}$ mesmo autor refere que à medida que 
a comunidade local adquire uma compreensão básica sobre aquilo que é o turismo as atitudes/ perceções da mesma vão-se alterando positivamente. Considera-se, assim, relevante o desenvolvimento de estudos que analisem quais as atitudes/perceções dos residentes em relação ao Turismo (nos destinos rurais), considerando as diferentes fases do modelo Buttler (1980), sobre o ciclo de desenvolvimento dos destinos turísticos.

A maioria dos estudos referiu que as redes são um fator bastante importante para o desenvolvimento sustentável do turismo em áreas rurais, porém apenas um dos estudos (Tolstad, 2014) analisou este tema em profundidade. Assim é relevante o desenvolvimento de outros estudos que permitam confirmar o poder que a criação de redes tem no desenvolvimento das empresas instaladas nestas áreas e das próprias áreas.

Por fim, a conclusão geral a que se chega após a análise dos artigos selecionados para este artigo, é a de que não existem estudos que analisem especificamente o papel das experiências turísticas no desenvolvimento sustentável do turismo e do destino nas áreas rurais, ou seja, de que forma a inclusão ou não de experiências melhoram/prejudicam o desenvolvimento turístico numa dada região rural, e consequente desenvolvimento sustentável do destino.

\section{Bibliografia}

Agapito, D., Valle, P., \& Mendes, J.

2012. "Sensory marketing and tourist experiences". Journal of Spatial and Organizational Dynamics, 10: 7-19.

Agapito, D., Valle, P., \& Mendes, J.

2014. "The sensory dimension of tourist experiences: Capturing meaningful sensory-informed themes in Southwest Portugal". Tourism Management, 42: 224-237.

Aho, S. K.

2001. "Towards a general theory of touristic experiences: Modelling experience process in tourism".

Tourism Review, 56(3/4): 33-37.

Andersson, T. D.

2007. "The tourist in the experience economy". Scandinavian Journal of Hospitality and Tourism, 7(1): 46-58.

Ballantyne, R., Packer, J., \& Sutherland, L. A.

2011. "Visitors' memories of wildlife tourism: Implications for the design of powerful interpretive experiences". Tourism Management, 32(4): 770-779.

Boo, S., \& Lu, X.

2015. "Tourist's world Expo experiences". Event Management, 19: 123-142.

Carneiro, M. J., Lima, J., \& Silva, A. L.

2015. "Landscape and the rural tourism experience: Identifying key elements, addressing potential, and implications for the future". Journal of Sustainable Tourism, 23(8-9): 1217-1235. https://doi. org/10.1080/09669582.2015.1037840

Carneiro, M. J., Lima, J., \& Silva, A. L.

2017. "Landscape and the rural tourism experience: Identifying key elements, addressing potential, and implications for the future". Journal of Sustainable Tourism, 23(8-9): 1217-1235.

Carvão, S.

2009. "Tendências do turismo internacional". Revista Exedra, 17-32.

Cebrián, F., \& Sánchez, I.

2016. "The landscape as a tourist resource and its impact in mountain areas in the south of Castilla-La-Mancha (Spain)". International Journal of Sustainable Development and Planning, 11(3): 345-354. Dissart, J., \& Marcouiller, D. W.

2012. "Rural tourism production and the experience-scape". Tourism Analysis, 17(6): 691-704.

Eusébio, C., Kastenholz, E., \& Breda, Z.

2014. "Tourism and sustainable development of rural destinations: A stakeholders' view". Revista Portuguesa de Estudos Regionais, 36(1): 13-21. 
Ezeuduji, I. O.

2015. "Strategic event-based rural tourism development for sub-Saharan Africa". Current Issues in Tourism, 318(3): 212-228.

Ezeuduji, I. O., \& Rid, W.

2011. "Rural tourism offer and local community participation in the Gambia". Tourismos, 6(2): 187-211.

Geus, S. De, Richards, G., \& Toepoel, V.

2016. "Conceptualisation and operationalisation of event and festival experiences: Creation of an event experience scale”. Scandinavian Journal of Hospitality and Tourism, 16(3): 274-296.

Holbrook, M. B., \& Hirschman, E. C.

1982. "The experiential aspects of consumption: Consumer fantasies, feelings, and fun". Journal of Consumer Research, 9(2): 132.

Hosany, S., \& Gilbert, D.

2010. "Measuring tourists' emotional experiences toward hedonic holiday destinations". Journal of Travel Research, 49(4): 513-526.

Hosany, S., \& Witham, M.

2009. "Dimensions of cruisers' experiences, satisfaction and intention to recommend". Journal of Travel Research, 49(3): 351-364.

Huijibens, E.

2012. "Tourist System (pp. 254-256)". In P. Robinson (Ed.), Tourism: The Key Concepts. London, United

Kingdom: Routledge. Please see APA style for book chapters

Idziak, W., Majewski, J., \& Zmyslony, P.

2015. "Community participation in sustainable rural tourism experience creation: A long-term appraisal and lessons from a thematic villages project in Poland". Journal of Sustainable Tourism, 23(8-9): 1341-1362.

Kastenholz, E., Carneiro, M. J., Marques, C. P., \& Lima, J.

2012. "Understanding and managing the rural tourism experience - The case of a historical village in Portugal". Tourism Management Perspectives, 4(0): 207-214.

Kim, J. H., Ritchie, J. R. B., \& McCormick, B.

2012. "Development of a scale to measure memorable tourism experiences". Journal of Travel \& Tourism Marketing, 51(1): 12-25.

Kim, S., \& Jamal, T.

2015. "The co-evolution of rural tourism and sustainable rural development in Hongdong, Korea : Complexity, conflict and local response". Journal of Sustainable Tourism, 23(8-9): 1363-1385. https:// doi.org/10.1080/09669582.2015.1022181

Knutson, B. J., \& Beck, J. A.

2008. "Identifying the dimensions of the experience construct: Development of the model". Journal of Quality Assurance in Hospitality \& Tourism, 4(3-4): 23-35.

Lee, H. M., \& Smith, S. L. J.

2015. "A visitor experience scale: Historic sites and museums". Journal of China Tourism Research, 11(3), 255-277. https://doi.org/10.1080/19388160.2015.1083499

Lepp, A.

2008. "Attitudes towards initial tourism development in a community with no prior tourism experience: The sase of Bigodi, Uganda". Journal of Sustainable Tourism, 16(1): 5-22. https://doi.org/10.2167/ jost630.0

Loureiro, M.

2014. "The role of the rural tourism experience economy in place attachment and behavioral intentions". International Journal of Hospitality Management, 40(1): 1-9. https://doi.org/10.1016/j.ijhm.2014.02.010

Lun, L., Pechlaner, H., \& Volgger, M.

2016. "Rural tourism development in mountain regions : Identifying success factors, challenges and potentials". Journal of Quality Assurance in Hospitality \& Tourism, 17(4): 389-411. https://doi.org /10.1080/1528008X.2015.1096754

Mehmetoglu, M., \& Engen, M.

2011. "Pine and Gilmore's concept of experience economy and its dimensions: An empirical examination in tourism". Journal of Quality Assurance in Hospitality \& Tourism, 12(4): 237-255. https://doi.org /10.1080/1528008X.2011.541847 
Morgan, M., \& Xu, F.

2010. "Student travel experiences: Memories and dreams (pp. 118-138)". En N. Scott, E. Laws \& P. Boksberger (Eds.), Marketing of Tourism Experiences. Oxon, United Kingdom: Routledge, . Please see APA style for book chapters

Mossberg, L.

2007. "A marketing approach to the tourist experience". Scandinavian Journal of Hospitality and Tourism, 7(1): 59-74.

Murphy, A., \& Williams, P. W.

1999. "Attracting Japanese tourists into the rural hinterland: Implications for rural development and planning". Tourism Management, 20(4): 487-499.

Oh, H., Fiore, A., \& Jeoung, M.

2007. "Measuring experience economy concepts: Tourism applications". Journal of Travel Research, 46: 119-132.

Otto, J. E., \& Ritchie, J. R. B.

1996. "The service experience in tourism". Tourism Management, 17(3): 165-174.

Pine, B. J., \& Gilmore, J. H.

1998. "Welcome to the experience economy". Harvard Business Review, 76: 97-105.

Quadri-felitti, D. L., \& Fiore, A. M.

2013. "Destination loyalty: Effects of wine tourists' experience, memories, and satisfaction on intentions".

Tourism and Hospitality Research, 13(1): 47-62. https://doi.org/10.1177/1467358413510017

Quadri-felitti, D., \& Fiore, A. M.

2016. "Wine tourism suppliers' and visitors' experiential priorities". International Journal of Contemporary

Hospitality Management, 28(2): 397-417. https://doi.org/10.1108/IJCHM-05-2014-0224

Quan, S., \& Wang, N.

2004. "Towards a structural model of the tourist experience: An illustration from food experiences in tourism". Tourism Management, 25: 297-305.

Schmitt, B.

1999. "Experiential marketing". Journal of Marketing Management, 15: 53-67.

Scott, N., Laws, E., \& Boksberger, P.

2009. "The marketing of hospitality and leisure experiences". Journal of Hospitality Marketing \& Management, 18(2-3): 99-110. https://doi.org/10.1080/19368620802590126

Sharpley, R., \& Stone, P.

2011. Tourist Experience - Contemporary Pespectives. Nova Iorque: Routledge.

Song, H. J., Ahn, Y., \& Lee, C.

2015. "Examining relationships among expo experiences, service quality, satisfaction, and the effect of the Expo: The case of the Expo 2012 Yeosu Korea". Asia Pacific Journal of Tourism Research, 20(11): 1266-1285. https://doi.org/10.1080/10941665.2014.965719

Suntikul, W., \& Jachna, T.

2016. "Profiling the heritage experience in Macao's historic center". International Journal of Tourism

Research, 18(4): 308-318. https://doi.org/10.1002/jtr

Swarbrooke, J., \& Horner, S.

2002. O Comportamento do Consumidor no Turismo. São Paulo: Aleph.

Tolstad, H. K.

2014. "Development of rural-tourism experiences through networking: An example from Gudbrandsdalen, Norway". Norsk Geografisk Tidsskrift - Norwegian Journal of Geography, 68(2): 111-120. https://doi. org/10.1080/00291951.2014.894561

Tung, V. W. S., \& Ritchie, J. R. B.

2011. "Exploring the essence of memorable tourism experiences". Annals of Tourism Research, 38(4): 1367-1386.

Walls, A. R., Okumus, F., Wang, Y. R., \& Kwun, D. J. W.

2011. "An epistemological view of consumer experiences". International Journal of Hospitality Management, 30(1): 10-21.

$\mathrm{Xu}$, J. B., \& Chan, A.

2010. "Service experience and package tours". Asia Pacific Journal of Tourism Research, 15(2): 177-194. https://doi.org/10.1080/10941661003629987 
Apêndice A. Lista de artigos considerados na primeira fase da análise (ordenados cronologicamente)

1. Oh, H., Fiore, A.M., \& Jeoung, M.

2007. "Measuring experience economy concepts: Tourism applications". Journal of Travel Research, 46 (2): 119-132.

2. Scott, N., Laws, E., \& Boksberger, P.

2009. "The marketing of hospitality and leisure experiences". Journal of Hospitality and Leisure Marketing, 18 (2-3): pp. 99-110.

3. Hosany, S., \& Witham, M.

2010. "Dimensions of cruisers' experiences, satisfaction, and intention to recommend". Journal of Travel Research, 49 (3): 351-364.

4. Xu, J., \& Chan, A.

2010. "Service experience and package tours". Asia Pacific Journal of Tourism Research, 15 (2): 177-194.

5. Mehmetoglu, M., \& Engen, M.

2011. "Pine and Gilmore's concept of experience economy and its dimensions: An empirical examination in tourism". Journal of Quality Assurance in Hospitality and Tourism, 12 (4): 237-255.

6. Kastenholz, E., Carneiro, M.J., Peixeira Marques, C., \& Lima, J.

2012. "Understanding and managing the rural tourism experience - The case of a historical village in Portugal". Tourism Management Perspectives, 4: 207-214.

7. Quadri-Felitti, D.L., \& Fiore, A.M.

2013. "Destination loyalty: Effects of wine tourists' experiences, memories, and satisfaction on intentions". Tourism and Hospitality Research, 13 (1): 47-62.

8. Loureiro, S.M.C.

2014. "The role of the rural tourism experience economy in place attachment and behavioral intentions". International Journal of Hospitality Management, 40: 1-9.

9. Agapito, D., Valle, P., \& Mendes, J.

2014. "The sensory dimension of tourist experiences: Capturing meaningful sensory-informed themes in Southwest Portugal". Tourism Management, 42: 224-237.

10. Song, H.J., Ahn, Y.J., \& Lee, C.K.

2015. "Examining relationships among Expo experiences, service quality, satisfaction, and the effect of the Expo: The case of the Expo Yeosu Korea". Asia Pacific Journal of Tourism Research, 20 (11): 1266-1285.

11. Lee, H.M., \& Smith, S.L.J.

2015. "A visitor experience scale: Historic sites and museums". Journal of China Tourism Research, 11 (3: 255-277.

12. Boo, S., \& Lu, X.

2015. "Tourists' world expo experiences". Event Management, 19 (1): 123-142.

13. Geus, S.D., Richards, G., \& Toepoel, V.

2016. "Conceptualisation and operationalisation of event and festival experiences: Creation of an event experience scale". Scandinavian Journal of Hospitality and Tourism, 16 (3): 274-296.

14. Quadri-Felitti, D., \& Fiore, A.M.

2016. "Wine tourism suppliers' and visitors' experiential priorities". International Journal of Contemporary Hospitality Management, 28 (2): 397-417.

15. Suntikul, W., \& Jachna, T.

2016. "Profiling the heritage experience in Macao's historic center". International Journal of Tourism Research, 18 (4): 308-318.

Apêndice B. Lista de artigos considerados na segunda fase da análise (ordenados cronologicamente)

1. Murphy, A., \& Williams, P.W.

1999. "Attracting Japanese tourists into the rural hinterland: Implications for rural development and planning". Tourism Management, 20 (4): 487-499.

2. Lepp, A.

2008. "Attitudes towards initial tourism development in a community with no prior tourism experience:

The case of Bigodi, Uganda". Journal of Sustainable Tourism, 16 (1): 5-22. 
3. Ezeuduji, I.O., \& Rid, W.

2011. "Rural tourism offer and local community participation in the Gambia". Tourismos, 6 (2): 187-211. 4. Dissart, J.C., \& Marcouiller, D.W.

2012. "Rural tourism production and the experience-scape". Tourism Analysis, 17 (6): 691-704.

5. Eusébio, C., Kastenholz, E., \& Breda, Z.

2014. "Tourism and sustainable development of rural destinations: A stakeholders' view". Revista

Portuguesa de Estudos Regionais, 36 (1): 13-21.

6. Tolstad, H.K.

2014. "Development of rural-tourism experiences through networking: An example from Gudbrandsdalen, Norway". Norsk Geografisk Tidsskrift, 68 (2): 111-120.

7. Carneiro, M.J., Lima, J., \& Silva, A.L.

2015. "Landscape and the rural tourism experience: Identifying key elements, addressing potential, and implications for the future". Journal of Sustainable Tourism, 23 (8-9): 215-1235.

8. Idziak, W., Majewski, J., \& Zmyślony, P.

2015. "Community participation in sustainable rural tourism experience creation: A long-term appraisal and lessons from a thematic villages project in Poland". Journal of Sustainable Tourism, 23 (8-9): $1341-1362$.

9. Ezeuduji, I.O.

2015. "Strategic event-based rural tourism development for sub-Saharan Africa". Current Issues in Tourism, 18 (3) 212-228.

10. Kim, S., \& Jamal, T.

2015. "The co-evolution of rural tourism and sustainable rural development in Hongdong, Korea: Complexity, conflict and local response". Journal of Sustainable Tourism, 23 (8-9): 1363-1385.

11. Lun, L.M., Pechlaner, H., \& Volgger, M.

2016. "Rural tourism development in mountain regions: Identifying success factors, challenges and potentials". Journal of Quality Assurance in Hospitality and Tourism, 17 (4): 389-411.

12. Cebrián, F., \& Sánchez, I.

2016. "The landscape as a tourist resource and its impact in mountain areas in the south of castilla-la mancha (Spain)". International Journal of Sustainable Development and Planning, 11 (3): 345-354. 